\title{
The early-type dwarf galaxy population of the Fornax cluster ${ }^{\star}$
}

\author{
S. Mieske ${ }^{1}$, M. Hilker ${ }^{2}$, L. Infante ${ }^{3}$, and C. Mendes de Oliveira ${ }^{4}$ \\ 1 European Southern Observatory, Karl-Schwarzschild-Strasse 2, 85748 Garching bei München, Germany \\ e-mail: smieske@eso.org \\ 2 Argelander Institut für Astronomie, Abteilung Sternwarte, Auf dem Hügel 71, 53121 Bonn, Germany \\ e-mail: mhilker@astro.uni-bonn.de \\ 3 Departamento de Astronomía y Astrofísica, Pontificia Universidad Católica de Chile, Casilla 306, Santiago 22, Chile \\ e-mail: linfante@astro.puc.cl \\ ${ }^{4}$ Instituto de Astronomia, Geofísica, e Ciências Atmosféricas, Departamento de Astronomia, Universidade de São Paulo, \\ Rua do Matáo 1226, Cidade Universitãria, 05508-900 São Paulo, SP, Brazil \\ e-mail: oliveira@astro.iag.usp.br
}

Received 25 August 2006 / Accepted 17 October 2006

\section{ABSTRACT}

\begin{abstract}
Aims. We analyse the photometric properties of the early-type Fornax cluster dwarf-galaxy population $\left(M_{\mathrm{V}}>-17\right.$ mag), based on a wide-field imaging study of the central cluster area in $V$ and $I$ bandpasses. We used the instrument/telescope combination IMACS/Magellan at Las Campanas Observatory, providing much larger light-collecting area and better image resolution than previous wide-field imaging surveys.

Methods. We created a fiducial sample of Fornax cluster dwarf ellipticals (dEs) in the following three steps. (1) To verify cluster membership, we measured $I$-band surface brightness fluctuations (SBF) distances to candidate dEs known from previous surveys. (2) We re-assessed morphological classifications for those candidate dEs that are too faint for SBF detection. (3) We searched for new candidate dEs in the size-luminosity regime close to the resolution limit of previous surveys.

Results. (1) We confirm cluster membership for 28 candidate dEs in the range $-16.6<M_{\mathrm{V}}<-10.1$ mag by means of SBF measurement. We find no SBF background galaxy. (2) Of 51 other candidate dEs in the range $-13.2<M_{\mathrm{V}}<-8.6 \mathrm{mag}, 2 / 3$ are confirmed as probable cluster members by morphological re-assessment, while $1 / 3$ are re-classified as probable background objects. (3) We find 12 new $\mathrm{dE}$ candidates in the range $-12.3<M_{\mathrm{V}}<-8.8 \mathrm{mag}$, two of which are directly confirmed via SBF measurement. The resulting fiducial dE sample follows a well-defined surface brightness-magnitude relation, showing that Fornax dEs are about $40 \%$ larger than Local Group dEs. The sample also defines a colour-magnitude relation that appears slightly shallower than that of Local Group dEs. The early-type dwarf galaxy luminosity function in Fornax has a very flat faint end slope $\alpha \simeq-1.1 \pm 0.1$. We discuss these findings in the context of structure-formation theories.

Conclusions. The SBF method is a very powerful tool to help constrain the faint end of the galaxy luminosity function in nearby galaxy clusters. For the Fornax cluster, morphological cluster memberships - if performed at sufficient resolution - are very reliable.
\end{abstract}

Key words. galaxies: clusters: individual: Fornax cluster - galaxies: dwarf - galaxies: fundamental parameters galaxies: luminosity function, mass function - techniques: photometric

\section{Introduction}

The Fornax cluster is the most prominent nearby galaxy cluster in the southern hemisphere, being located at about $19 \mathrm{Mpc}$ $((m-M)=31.39 \mathrm{mag})$ distance (Freedman et al. 2001). Due to its compact nature and the dominance of early-type galaxies (Ferguson 1989), the Fornax cluster is well suited to characterising the global properties of its galaxy population. The reference source of information on the Fornax galaxy population is the Fornax Cluster Catalog (FCC, Ferguson 1989), a wide-field imaging survey with $1.68^{\prime \prime}$ pixel scale on photographic scans that cover the inner $3.5^{\circ}$ of the cluster.

One of the most important quantities for characterising a galaxy population is the galaxy luminosity function (GLF). Its logarithmic faint-end slope $\alpha$ is a very useful quantity to be contrasted with the expected slope for the mass spectrum of cosmological dark-matter halos (e.g. Jenkins et al. 2001;

* Table 1 is only available in electronic form at the CDS via anonymous ftp to cdsarc.u-strasbg.fr (130.79.128.5) or via http://cdsweb.u-strasbg.fr/cgi-bin/qcat?J/A+A/463/503
Moore et al. 1999). Generally, the value of $\alpha$ derived in various environments including the Local Group is much shallower than the expected slope of dark matter halos (see for example Grebel et al. 2003; Trentham \& Tully 2002; Trentham et al. 2005; Andreon et al. 2006; Tanaka et al. 2005; and Infante et al. 2003 , and references therein). This discrepancy is also known as the "substructure problem" of present-day cosmology.

Up to now, investigations of the Fornax GLF in the low luminosity regime $\left(M_{\mathrm{V}}>-14 \mathrm{mag}\right)$ have been restricted to morphological cluster-membership assignment (e.g. Caldwell 1987; Ferguson \& Sandage 1988; Ferguson 1989; Phillipps et al. 1987; Kambas et al. 2000; Hilker et al. 2003). This is because spectroscopic surveys have not had the depth needed to obtain velocities for dE candidates fainter than about $\mu_{\mathrm{V}, 0} \simeq 23 \mathrm{mag} / \mathrm{arcsec}^{2}$ (e.g. Hilker et al. 1999a; Drinkwater et al. 2001). The faint end slopes derived for the Fornax GLF in the literature generally cover the range $-1.5<\alpha<-1.0$, depending on the magnitude limits and galaxy types considered. The important restriction of the morphological assessment is the uncertainty in estimating the amount of contamination by background galaxies (e.g. 
Trentham \& Tully 2002). This can lead to different authors deriving very different slopes for the same cluster: Ferguson \& Sandage (1988) obtain $\alpha=-1.08 \pm 0.09$ for the dwarf GLF in Fornax; Kambas et al. (2000) suggest a much steeper slope $\alpha \simeq-2.0$, based on poorer resolution data of $2.3^{\prime \prime}$ without colour information (see also the discussion in Hilker et al. 2003). Both surveys have comparable completeness limits of $M_{B} \simeq-12 \mathrm{mag}$. Such differences in $\alpha$ stress the need for high-resolution imaging and an extension of the limiting magnitude for direct cluster membership determination.

A note on nomenclature: for simplicity, throughout this paper we use the term $\mathrm{dE}$ (dwarf elliptical) to refer to early-type dwarf galaxies $\left(M_{\mathrm{V}}>-17 \mathrm{mag}\right)$ in general; i.e. the term $\mathrm{dE}$ also encompasses dS0 and dSph.

In Mieske et al. (2003, Paper I hereafter) we used Monte Carlo simulations to investigate the potential of the surfacebrightness-fluctuation (SBF) method (Tonry \& Schneider 1988) to directly determine cluster memberships of faint candidate dEs in nearby galaxy clusters. We find that with 1 -h $I$-band exposures on $8 \mathrm{~m}$ class telescopes and with good seeing $\left(0.5^{\prime \prime}\right)$, reliable SBF cluster memberships can be determined down to $M_{\mathrm{V}} \simeq-11 \mathrm{mag}$ at a $20 \mathrm{Mpc}$ distance. This is several magnitudes fainter than the limit in previous spectroscopic surveys.

In Hilker et al. (2003, Paper II hereafter) we present a widefield photometric study of the Fornax galaxy population, based on data obtained with the WFCCD camera at the $2.5 \mathrm{~m}$ duPont telescope at Las Campanas Observatory, Chile (LCO). This camera has a field of view of $25^{\prime}$ and a pixel scale of $0.8^{\prime \prime}$, enabling the detection of very faint $\mathrm{dE}$ candidates. Using a combination of visual inspection and SExtractor (Bertin \& Arnout 1996) automated object detection, we discovered about $70 \mathrm{dE}$ candidates in Fornax with $-12.7<M_{\mathrm{V}}<-8.5 \mathrm{mag}$, extending the FCC sample of Ferguson (1989) to about three magnitudes fainter. For constructing the GLF from the WFCCD imaging, we used the galaxies listed in the FCC as likely cluster members plus the newly discovered fainter $\mathrm{dE}$ candidates. As an ad-hoc correction for possible interlopers, we restricted the sample to galaxies within $2 \sigma$ of the colour-magnitude and surface brightnessmagnitude relation defined by the entire sample. The faint end slope derived from this was $\alpha=-1.10 \pm 0.10$, in good agreement with the value found by Ferguson \& Sandage (1988). Note that the error of this value is only statistical and does not account for systematic uncertainties of morphological classifications.

There were two deficits with the WFCCD data set, related to its large pixel scale of $0.8^{\prime \prime}$ and correspondingly large point source $F W H M$ of about $1.9^{\prime \prime}(\simeq 170$ pc at the Fornax cluster distance). 1. These data did not allow us to measure SBF amplitudes and hence derive direct cluster memberships for candidate dEs. 2. For $M_{\mathrm{V}}>-12 \mathrm{mag}$, the image resolution approached the expected size of Local Group (LG) dE analoga, with typical LG dEs being about twice as large as the seeing FWHM (see Fig. 1). Therefore, the data did not allow us to morphologically separate probable cluster members from background galaxies in that magnitude regime. Note that this resolution restriction applies even more to previous surveys such as the FCC or the Kambas et al. (2000) work.

In this paper we investigate the photometric properties of the dwarf galaxy population in the Fornax cluster $\left(M_{\mathrm{V}}>-17 \mathrm{mag}\right)$, following up on our Paper II study. We use data obtained with the instrument IMACS mounted at the $6.5 \mathrm{~m}$ Magellan telescopes at LCO, which provides about a four times smaller pixel scale and seven times larger light collecting area as compared to the WFCCD data of Paper II, as well as a comparable field of view. In Mieske et al. (2006b, Paper III hereafter), those data were

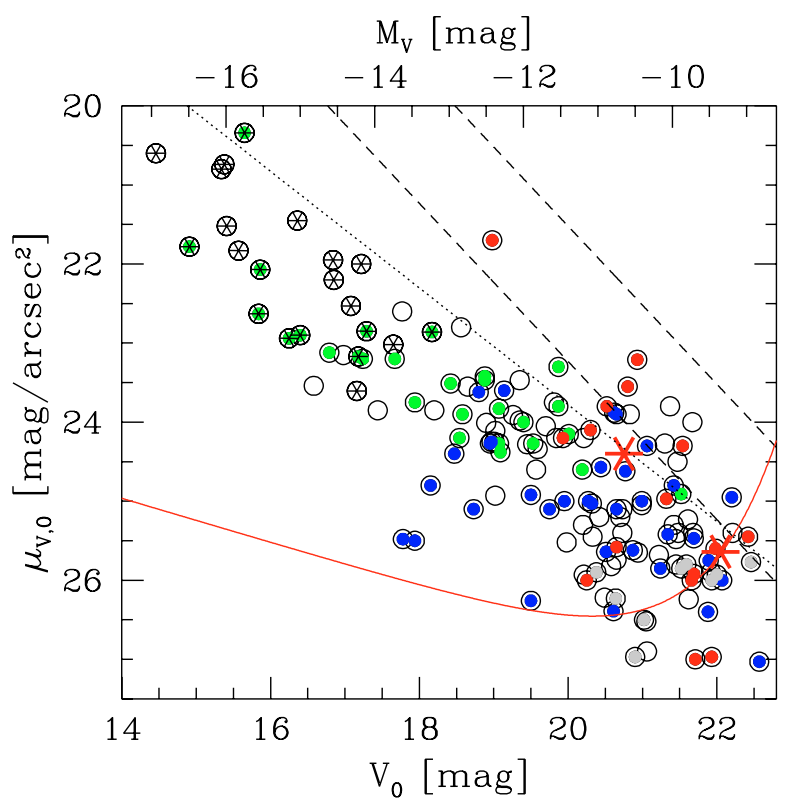

Fig. 1. Central surface brightness $\mu_{\mathrm{V}, 0}$ plotted vs. $V_{0}$ of the candidate dEs from Hilker et al. (2003, Paper II), based on WFCCD data. The solid line indicates the $50 \%$ completeness limit. The two large red asterisks indicate two candidate dEs found in a background WFCCD field. Small black asterisks indicate galaxies with confirmed cluster membership from radial velocity (Drinkwater et al. 2001; Mieske et al. 2004; Hilker et al. 1999a). Coloured circles indicate objects re-observed with the higher resolution IMACS data presented in this paper. See Fig. 2 for a map of the imaged Fornax cluster region. Green filled circles indicate galaxies with cluster membership confirmed from SBF (Sect. 3). Blue filled circles indicate galaxies with probable cluster membership based on revised morphology assessment (Sect. 4). Red filled circles indicate probable background galaxies based on revised morphological assessment (Sect. 4). Grey filled circles indicate unclear classifications (Sect. 4). The dotted line is a fit to the $M_{\mathrm{V}}-\mu_{\mathrm{V}, 0}$ values of Local Group dEs (Grebel et al. 2003). The lower dashed line indicates the location of an exponential galaxy light profile with $F W H M=3.5^{\prime \prime}$, which is twice the seeing for the Paper II data. The upper dashed line indicates an exponential profile with $F W H M=1.5^{\prime \prime}$, which is about twice the seeing in the IMACS data presented in this paper.

already presented and have been used to derive a calibration of the SBF method at blue colours.

The IMACS data are presented in Sect. 2. In the subsequent three chapters we describe the following steps to compile a fiducial Fornax dE sample:

1. Confirm cluster membership of candidate dEs by means of $I$-band SBF measurement in Sect. 3.

2. Re-assess the morphological classification of candidate dEs too faint for SBF detection in Sect. 4.

3. Search for new candidate dEs close to the resolution limit of previous surveys in Sect. 5.

The photometric scaling relations and luminosity function of the resulting fiducial Fornax dwarf galaxy population are discussed in Sect. 6. In Sect. 7 we finish this paper with a summary and conclusions.

\section{The data}

The imaging data for this paper were obtained with the instrument IMACS at Las Campanas Observatory, Chile (see also Paper III). Using the "short" $\mathrm{f} / 2$ camera, a 27.4" field was imaged 


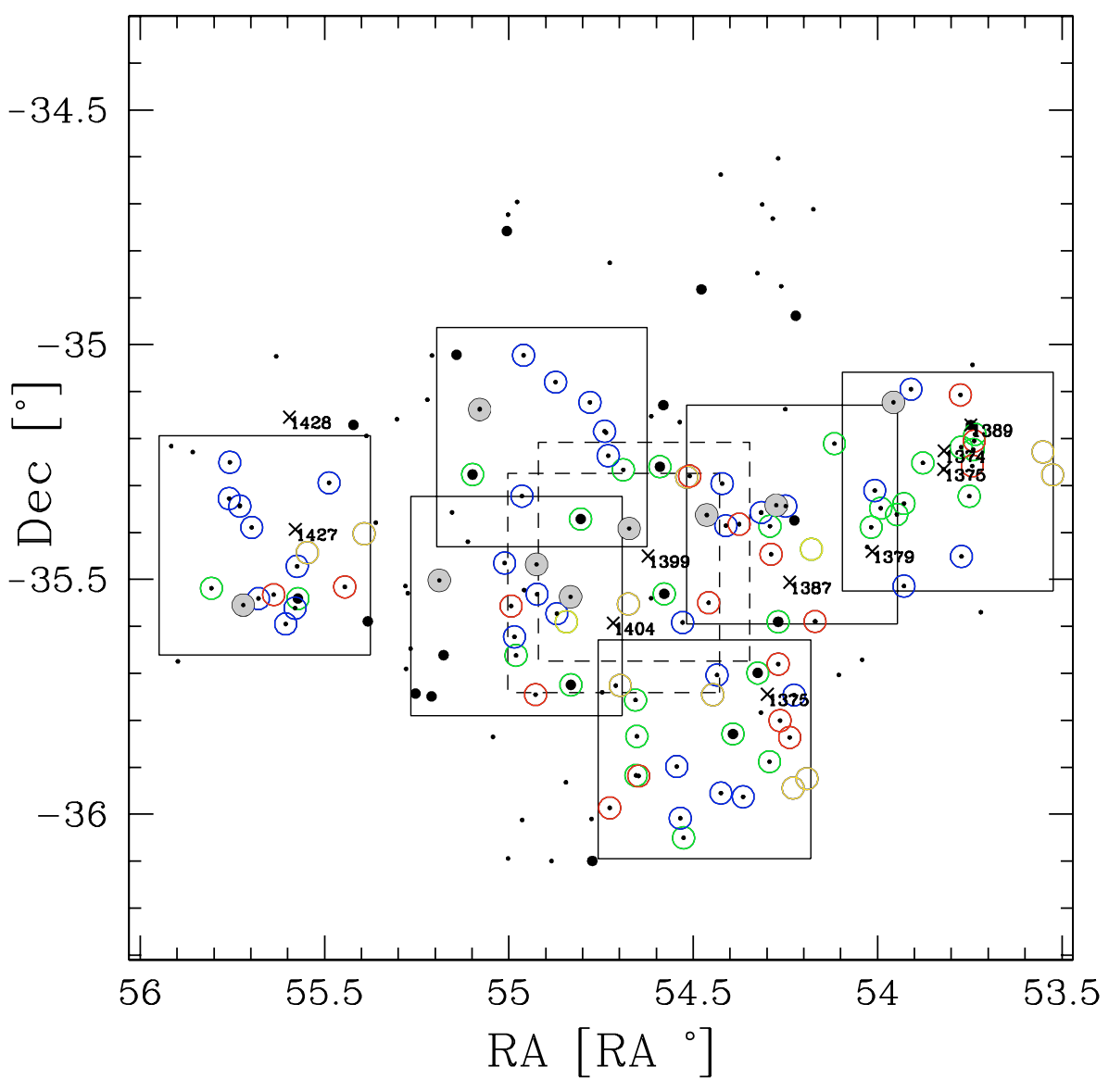

Fig. 2. Map of the central Fornax cluster. Small dots are cluster member candidates from Hilker et al. (2003, Paper II). Large dots are cluster members previously confirmed by radial velocity (Drinkwater et al. 2001; Mieske et al. 2004; Hilker et al. 1999a). Squares indicate the IMACS pointings from this paper. The two dashed squares indicate the two central IMACS pointings with lower integration times (see text). The coloured circles mark objects observed within those pointings. Green circles are cluster members confirmed by SBF (Sect. 3). Blue circles are probable members based on a morphological analysis (Sect. 4). Red circles are probable background galaxies based on a morphological analysis (Sect. 4). Grey filled circles are unclear classifications (Sect. 4). Golden circles without a dot inside indicate the new $\mathrm{dE}$ candidates detected with the IMACS imaging (Sect. 5). Those candidates from Paper II that were not observed were either outside the vignetted field of view or fell in the gaps between chips. Crosses mark the giant Fornax galaxies with their corresponding NGC numbers indicated. onto eight $2 \mathrm{k} \times 4 \mathrm{k}$ chips which have a pixel scale of $0.2^{\prime \prime}$. Seven fields in the central Fornax cluster were observed in both $V$ and $I$ under photometric conditions, covering the inner square degree ( $\simeq 330 \mathrm{kpc})$ and slightly beyond (see Fig. 2$)$. The total I-band integration time was between 3800 and $5160 \mathrm{~s}$, except for the central NGC 1399 field, which had $900 \mathrm{~s}$ integration for each of the two dithered exposures. The total integration times in $V$ were between 1200 and 1800 s except for the central field around NGC 1399, for which it was $900 \mathrm{~s}$ for each of the two slightly dithered exposures. The main part of the observing time was used for the $I$-band in order to measure SBF in this filter. Table 1 lists the candidate dEs from Paper II that were imaged with IMACS. This table gives their photometric parameters and coordinates. It also indicates their cluster membership flags that will be explained in Sects. 3 to 5 .

The image reduction before SBF measurement was done in the following steps: first, a master-bias was created for each chip and was subtracted from the domeflat exposures. Then for each chip the bias corrected dome flats were combined to create master-domeflats. Having the master-biases and masterdomeflats prepared for each chip, we used the COSMOS package $^{1}$ to do bias-subtraction, trimming and flat-field correction of the raw science frames. The reduced single science frames were registered with integer pixel shifts to avoid distortion of the SBF power spectrum and combined using a min-max rejection algorithm. The seeing $F W H M$ of the combined images typically ranged between 0.6 and $1.2^{\prime \prime}$, with a median around $0.8^{\prime \prime}$.

${ }^{1}$ Carnegie Observatories System for MultiObject Spectroscopy, http://llama.lco.cl/ aoemler/COSMOS.html

\section{Direct cluster membership assignment via SBF measurement}

The SBF measurements for the candidate dEs were performed in the $I$-band and are described in detail in Paper III. The signal-tonoise ratio of the SBF measurement is defined as $S / N=\frac{P_{0}}{P_{1}}$, where $P_{0}$ is the amplitude of the proper stellar SBF signal, while $P_{1}$ is the white noise amplitude. The amplitude of the background SBF fluctuations (arising from undetected intracluster globular clusters, background galaxies, and CCD effects like fringing) ranged between 0.1 and $0.55 \mathrm{mag}$.

We classified as confirmed cluster members all galaxies for which the SBF measurement had a $S / N>3$, see Table 1 of Paper III. All those 25 galaxies have SBF distances compatible to within $1.9 \sigma$ with the Fornax cluster reference distance of $31.39 \pm 0.12 \mathrm{mag}$ when applying the calibration D from Paper III. This specific calibration implies a bifurcated relation between colour $(V-I)_{0}$ and absolute fluctuation magnitude $\bar{M}_{\mathrm{I}}$. In Paper III it was marginally favoured over a non-bifurcated relation at the $1.8 \sigma$ level. When opting for a non-bifurcated relation instead, the scatter of the implied SBF distances increases naturally (see Tables 2 and 3 of Paper III). In that case, one galaxy is attributed a very low distance more than $3 \sigma$ below the reference distance, namely FCC 218 with $(m-M)=$ $30.32 \pm 0.33 \mathrm{mag}$. For its magnitude, this galaxy is not an outlier in terms of colour or surface brightness, see Table 1 and Sect. 6. Including it in the sample of Fornax cluster members therefore does not influence the statements made further on in this paper.

The 25 galaxies with SBF confirmed cluster membership span the range $-16.6<M_{\mathrm{V}}<-11.2 \mathrm{mag}$. Those galaxies are assigned a membership flag 1 in Table 1 of this paper (see also 


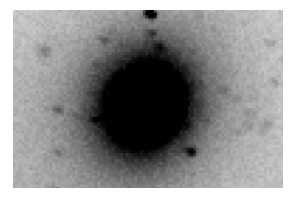

FCC 222

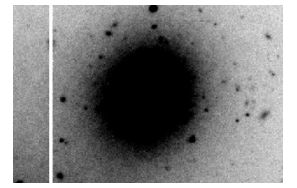

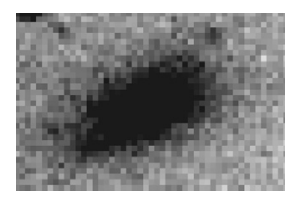

FCC 196

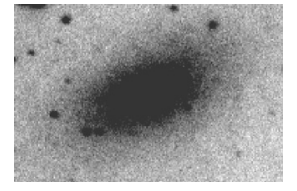

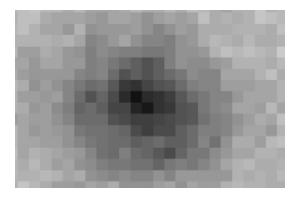

FCC 191

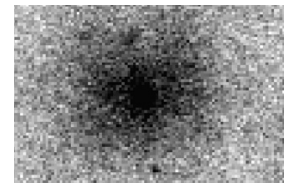

Fig. 3. Thumbnails of SBF-confirmed Fornax cluster members (Sect. 3), i.e. those with flag $=1$ in Table 1. Top: WFCCD images. The PSF FWHM is about $1.8^{\prime \prime}$. Bottom: IMACS images. The PSF FWHM is about $0.8^{\prime \prime}$. The thumbnail sizes are from left to right $77 \times 50^{\prime \prime}(7 \times$ $4.6 \mathrm{kpc}$ at the Fornax cluster distance), $38 \times 25^{\prime \prime}(3.5 \times 2.3 \mathrm{kpc})$, and $19 \times 12^{\prime \prime}(1.8 \times 1.1 \mathrm{kpc})$.

Fig. 3 for example thumbnail images). There are 7 additional galaxies for which an SBF signal with $S / N<3$ was detected. Three of them have background SBF below $50 \%$ of the stellar SBF signal and an SBF distance error below 0.5 mag. We include those three sources in the sample of confirmed cluster members assigning a flag value of 1.3. This yields a full sample of 28 galaxies spanning a luminosity range $-16.6<M_{\mathrm{V}}<$ $-10.1 \mathrm{mag}$. The remaining four dEs $\left(-12.4<M_{\mathrm{V}}<-10.4 \mathrm{mag}\right)$ with larger background fluctuations and SBF distance errors were defined as probable members from morphology with a membership flag of 1.7 (see also the next section). Note that nine of the $28 \mathrm{SBF}$ confirmed members also have radial velocity measurements available (Drinkwater et al. 2001), all of which are consistent with them being cluster members.

\section{Morphological re-classification}

In addition to allowing for SBF measurements, the IMACS data enable us to check which $\mathrm{dE}$ candidates from Paper II retain a smooth morphology when imaged at 2-3 times better spatial resolution.

For this morphological re-assessment, we defined three possible cases. i) The galaxy retains its smoothness on the IMACS data, so it is confirmed as probable cluster member (flag 2 in Table 1). ii) The galaxy exhibits clear substructure that is indicative of a background spiral, or the galaxy resolves into separate point sources (flag 3 in Table 1). Note that for the latter case we also demand that there is no low surface brightness envelope. Such an envelope would be indicative of a dwarf irregular galaxy (dIrr) that may be in the cluster. We did in any case not detect any such possible dIrr in our image inspections. iii) The IMACS data do not allow clear assessment (flag 4 in Table 1). The latter case iii) is restricted to very low surface brightness candidates (Fig. 1). Figures 4 and 5 show example cases for the morphological re-assessment. In the magnitude-surface brightness plot in Fig. 1, we indicate the respective classifications of the re-observed objects by different colour codings. We note that this morphological classification implicitly exploits the fact that the dwarf galaxy population in the central Fornax cluster is vastly dominated by early-type galaxies (Ferguson 1989).

The majority of candidate dEs are morphologically confirmed as probable cluster members: out of 60 candidate dEs with revised morphological assessment (and no SBF
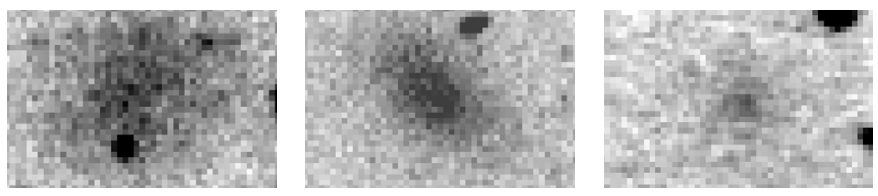
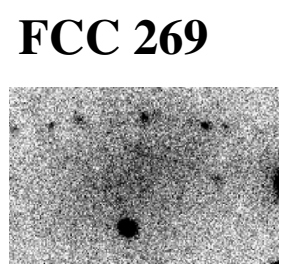
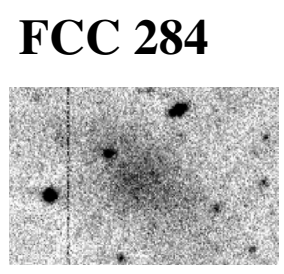

\section{WFLSB2-1}

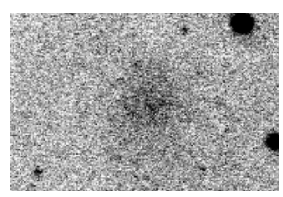

Fig. 4. Thumbnails of morphologically-confirmed Fornax cluster members (Sect. 4), i.e. those with flag $=2$ in Table 1. Top: WFCCD images. Bottom: IMACS images. The thumbnail sizes are $38 \times 25^{\prime \prime}(3.5 \times 2.3 \mathrm{kpc}$ at the Fornax cluster distance).

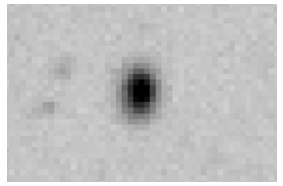

FCC 141

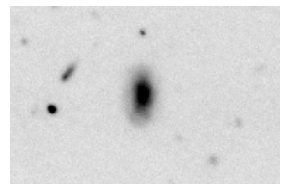

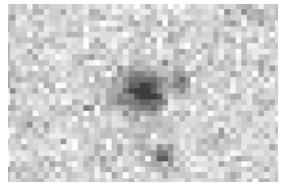

WFLSB 6-3

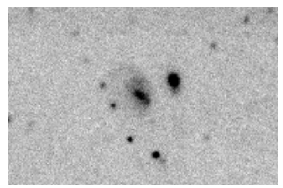

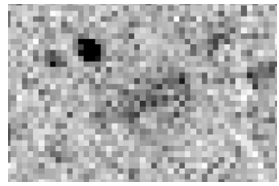
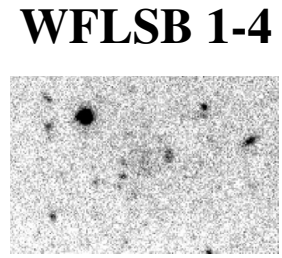

Fig. 5. Left two thumbnails: two morphologically re-classified background galaxies (Sect. 4), i.e. those with flag $=3$ in Table 1. Right thumbnail: a source with unclear classification, i.e. those with flag $=4$ in Table 1. Top: WFCCD images. Bottom: IMACS images. The thumbnail sizes are $38 \times 25^{\prime \prime}(3.5 \times 2.3 \mathrm{kpc}$ at the Fornax cluster distance).

measurement) in the range $-13.2<M_{\mathrm{V}}<-8.8$ mag, 34 are confirmed as probable members, 17 are re-classified as probable background, and 9 have unclear definitions. The contamination by background galaxies is thus about $1 / 3$ (17 out of 51). However, for calculating the GLF in Paper II we excluded galaxies more than $2 \sigma$ outside the mean surface-brightness magnitude relation to reduce the sample contamination. When considering only candidates within the $2 \sigma$ surface brightness limit and brighter than the $50 \%$ detection limit of $M_{\mathrm{V}}=-9.8 \mathrm{mag}$ from Paper II, the contamination drops to about $20 \%$. Figure 1 shows that as expected, the sources re-classified as background galaxies lie close to the resolution limit of the WFCCD data. We finally note that the WFCCD data from Paper II included one pointing towards an empty comparison field outside the Fornax cluster in which two candidate dEs were detected (see Fig. 1). Given that the average number of candidate dEs per pointing in Fornax was $6.5 \pm 0.7$ (Paper II), the formal background contamination derived from the detection of these two sources is in the range $10 \%$ to $50 \%$. This is consistent with the amount of contamination estimated from the IMACS morphological re-assessment.

Apart from morphological re-classification, we have also re-measured surface brightness profiles, total magnitudes and colours for the Paper II candidate dEs. We describe these measurements in detail in Sect. 6, but mention this re-measurement 


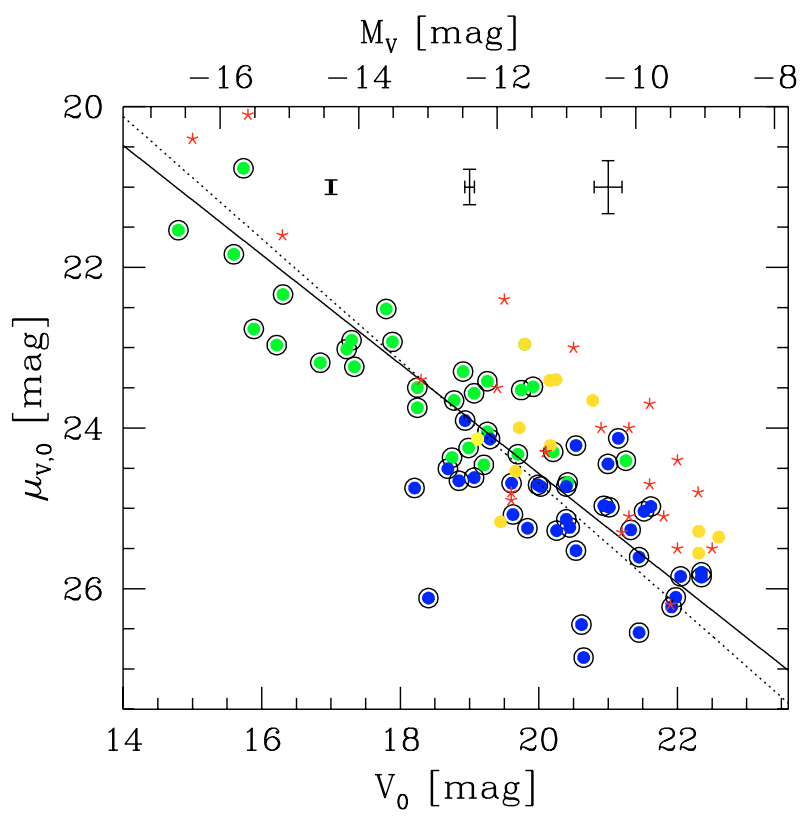

Fig. 6. Magnitude-surface brightness plot as in Fig. 1, now using the new photometry of the IMACS data and plotting only probable cluster members from SBF memberships and morphology (Sects. 3 and 4). Typical error bars are indicated. The magnitudes were redenningcorrected using Schlegel et al. (1998). The golden dots indicate newly found $\mathrm{dE}$ candidates in the IMACS data (Sect. 5). The solid line is a fit to the data points applying a $3 \sigma$ clipping. The dotted line shows the fit from the WFCCD photometry of all $\mathrm{dE}$ candidates from Hilker et al. (2003, Paper II), also applying a $3 \sigma$ clipping. The red asterisks indicate Local Group dEs (Grebel et al. 2003).

here since in the next subsection we refer to the revised surface brightness-magnitude plot in Fig. 6.

\subsection{SBF background galaxies?}

Do some galaxies in our sample qualify as SBF-confirmed background galaxies? This would be the case for galaxies without an SBF signal that are bright and large enough to expect such a signal at the Fornax distance. In that context it is striking that all morphologically-selected cluster members for which no SBF signal was detected had faint surface brightnesses $\mu_{\mathrm{V}, 0} \geq$ $24 \mathrm{mag} / \operatorname{arcsec}^{2}$ (see Fig. 6). There is only a small overlap region in surface brightness $\left(24<\mu_{\mathrm{V}, 0}<24.6 \mathrm{mag} / \operatorname{arcsec}^{2}\right)$ where both SBF memberships and morphological memberships are assigned. Galaxies in this overlap region with no detected SBF signal are the only ones that could in principle be confirmed as SBF background galaxies. Of course, one also expects such an overlap even if all galaxies are cluster members. This is because of differing SBF detection limits among the galaxies, depending on observing conditions (seeing, integration time) and intrinsic SBF amplitudes. The overall surface brightness limit for SBF detection in our data is $\simeq 24.3 \mathrm{mag} / \operatorname{arcsec}^{2}$ (except for the central pointing with shorter integration time). This limit is between the estimates derived in Paper I for seeing FWHM of $0.5^{\prime \prime}$ and $1.0^{\prime \prime}$ and at comparable integration times, which fits the fact that the median seeing of our data was $0.8^{\prime \prime}$.

In the following, we briefly discuss the galaxies in the overlap surface brightness region. The detectability of the SBF signal decreases at a fixed surface brightness when going to galaxies with fainter total luminosities, given that the SBF sampling area decreases. This fact can explain the occurrence

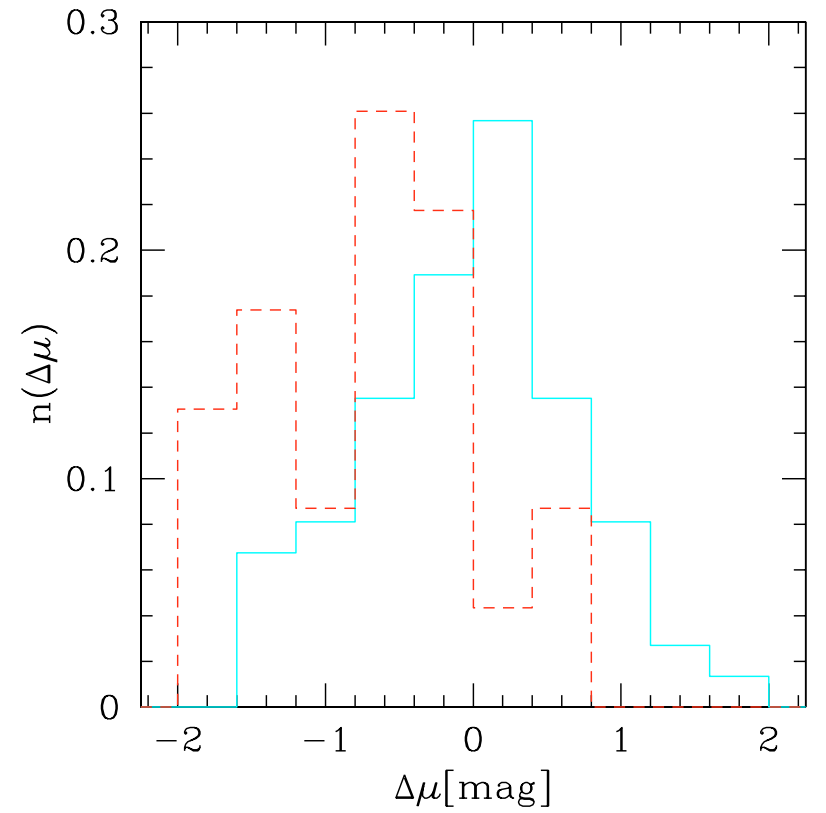

Fig. 7. This plot shows the normalised distribution of central surface brightnesses of the Fornax sample (solid histogram) and Local Group sample (dashed histogram) relative to the surface-brightness magnitude relation in Fornax (Eq. (1)). By definition, the Fornax distribution is centred on 0 . This plot shows that the overall shift in surface brightness between Fornax and Local Group is due to both a lack of small and overabundance of large galaxies in Fornax. According to a KS test, both distributions share the same parent distribution at only $0.08 \%$ probability. When shifting the Local Group distribution such that the mean of both distributions agree, the KS probability is $99.9 \%$.

of 3 galaxies with only morphological membership in the range $20.5<V<21.5 \mathrm{mag}, 24<\mu_{\mathrm{V}, 0}<24.6 \mathrm{mag}$ (Fig. 6). We find two more galaxies in the same $\mu_{\mathrm{V}, 0}$ range but about $1.5 \mathrm{mag}$ brighter. These are indeed the only two sources from this plot that might qualify as SBF confirmed background galaxies. The brighter of these galaxies is FCC 197. It actually had an SBF signal consistent with the cluster distance, but it is one of those four sources whose $S / N$ was too low and background fluctuation too large to reliably classify it as an SBF member (flag $=1.7$ in Table 1). The fainter galaxy is FCC 220. For this galaxy we did not detect a measurable SBF signal. The corresponding galaxy image has comparably bad seeing $\left(\simeq 0.9^{\prime \prime}\right)$, and the galaxy is quite red $((V-I) \simeq 1.14)$. These two facts decrease the detectability of the SBF signal. FCC 220 is furthermore only a few tenths of magnitudes brighter than the approximate limiting surface brightness for SBF detection in Fig. 6.

We therefore conclude that for none of the galaxies, the lack of a detectable SBF signal is enough to classify them as background.

\section{Search for new members}

The WFCCD data from Paper II had a resolution limit that is close to the intrinsic size of $\mathrm{LG} \mathrm{dEs}$ for $M_{\mathrm{V}}>-12$ mag (see also Fig. 1). The IMACS data now allow us to check how many $\mathrm{dE}$ candidates may have been overlooked in the WFCCD data because of this restriction.

To do so, we first added several hundred images of artificial dEs with colour $(V-I)=1.0$ in the range $-12.5<M_{\mathrm{V}}<$ $-10 \mathrm{mag}$ on top of the IMACS $I$-band images. For simulating them we assumed exponential surface brightness profiles. 

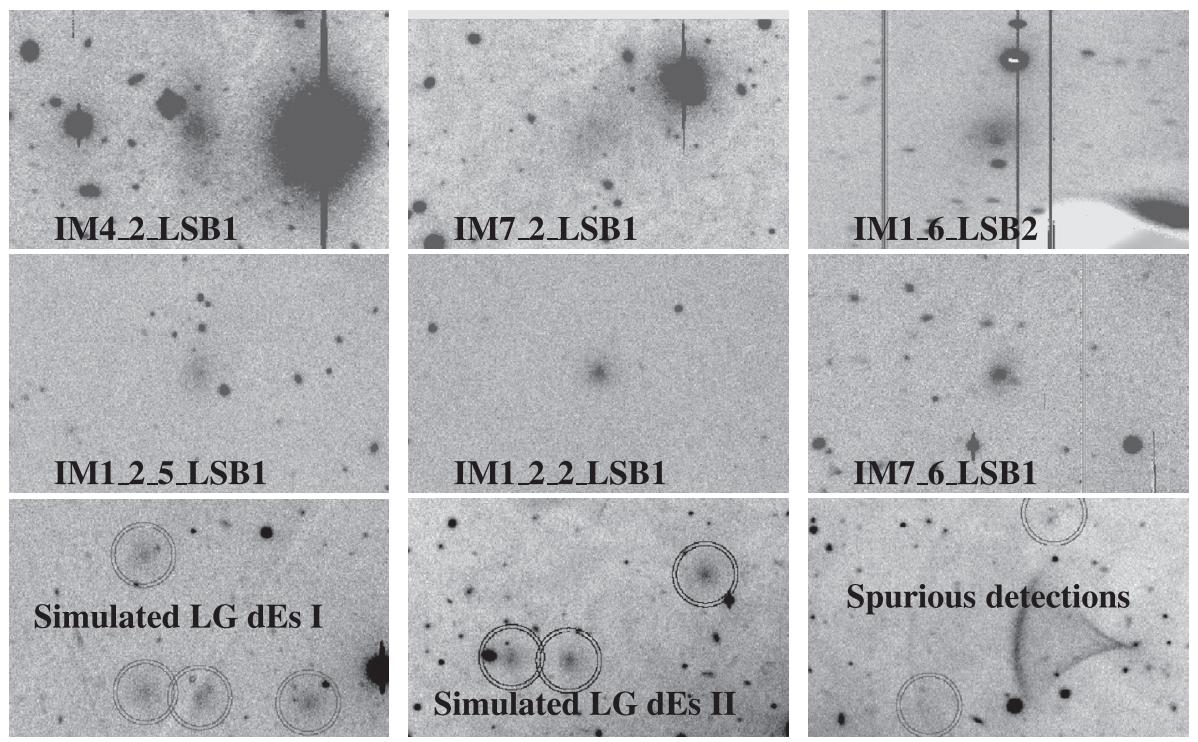

Fig. 8. Top two rows: example thumbnails for six of the 12 new Fornax dE candidates detected from our IMACS photometry (Sect. 5), see also Table 1 . Thumbnails are $82 \times 72^{\prime \prime}$ large $(7.5 \times 6.6 \mathrm{kpc}$ at the Fornax cluster distance). They are ordered from left to right and top to bottom by decreasing galaxy luminosity. Bottom row: the left thumbnail shows four simulated $\mathrm{dE}$ galaxies in the magnitude range $-10.8>M_{\mathrm{V}}>-11 \mathrm{mag}$ that have sizes corresponding to the LG dEs (simulated seeing $F W H M$ was $0.9^{\prime \prime}$ ). The middle thumbnail shows three simulated dEs in the same absolute magnitude range, but with only $60 \%$ the size of LG dEs. They are still clearly resolved. The right thumbnail shows two SExtractor detections that were not accepted as new $\mathrm{dE}$ candidates, one since it was part of a ghost image, another one because it was a blend of several very small sources.

Their central surface brightnesses were adopted to randomly scatter $\pm 1.5 \mathrm{mag}$ around the LG magnitude-surface brightness relation (Grebel et al. 2003; see also Fig. 1). This scatter corresponds to the $2 \sigma$ width of the magnitude-surface brightness relation for the Fornax $\mathrm{dE}$ candidates found in Paper II. We then let SExtractor run on those images to recover the simulated $\mathrm{dEs}$. To achieve efficient detection of those artifical galaxies and minimize the contamination by more compact sources, we demanded 400 connected pixels above $0.7 \sigma$ of the sky noise as detection threshold. We demanded 200 connected pixels only for the central pointing with lower integration time. The detection completeness ranged between $88 \%$ for the brightest dEs with $-12.5<M_{\mathrm{V}}<-12 \mathrm{mag}$ to $74 \%$ for the faintest dEs with $-10.5<M_{\mathrm{V}}<-10 \mathrm{mag}$, with an average of $80 \%$.

We then examined the SExtractor parameter space covered by those artificial dEs in terms of isophotal magnitude, surface brightness, Kron-radius, image area, and FWHM. To find new $\mathrm{dE}$ candidates, we run SExtractor on all IMACS images, applying the same detection parameters as for the IMACS images with added artificial galaxies. We then restricted the SExtractor output catalog to the parameter space recovered by the program for the artifical dEs. This selection was very efficient, resulting in only very few candidates per chip, sometimes none. We then compared those detected sources morphologically with the simulated $\mathrm{dE}$. We rejected obvious backgound objects like spirals, unresolved sources in the region of strong image distortion, fringing and reflection artefacts, and also groups of separate neighbouring sources that had not been correctly deblended by SExctractor (see Fig. 8 for examples). We also double-checked the image morphology in the shorter exposure $V$-band images. Obvious $V$-band dropouts - hence high $z$ objects - were rejected, although those were very few galaxies, all restricted to compact, relatively high-surface brightness objects. In addition to the automated SExtractor search, we finally inspected the images visually to search for sources morphologically representing the simulated LG dE analogs.

This search resulted in the detection of $12 \mathrm{dE}$ candidates in the range $-12.3<M_{\mathrm{V}}<-8.8 \mathrm{mag}$ that had not been detected in the WFCCD data in Paper II. Example images are shown in Fig. 8. Eight of the 12 new candidates were detected by SExtractor, and four additional ones by visual inspection only (see Table 1). This fraction of visual detections is consistent with SExtractor's incompleteness as quoted above. The locations of all new dE candidates are indicated in the Fornax map (Fig. 2). Their photometric parameters are listed in Table 1 and shown graphically in Figs. 6 and 9. Four of these $12 \mathrm{dE}$ candidates are located outside the image borders of the WFCCD data, all of which are included in the FCC as likely cluster members (see Table 1). There is furthermore one new $\mathrm{dE}$ candidate listed as a probable background galaxy in the FCC (see Table 1).

How many of the new $\mathrm{dE}$ candidates can be confirmed via SBF? We detected an SBF signal for only two galaxies, sources IM4_2_LSB1 and IM1_2_2_LSB1. The first galaxy has the highest total luminosity of the new $\mathrm{dE}$ candidates, while the second galaxy has the highest surface brightness. We applied the SBF reduction procedure from Paper III to these galaxies. The resulting SBF parameters are listed in Table 2. In that table, $B G$ gives the fraction of sky background fluctuation present in the uncorrected original fluctuation image. $\Delta_{\mathrm{GC}}$ gives the contribution of undetected GCs to the total flucutation signal in the original fluctuation image. The calibration relation adopted is the steep branch of case D in paper III, which is identical to the calibration by Tonry et al. (2001). The $S / N$ of the SBF signal was 5.0 for IM4_2_LSB1 and 1.9 for IM1_2_2_LSB1. Their SBF distances are marginally consistent with the Fornax cluster distance of 31.39 mag (Freedman et al. 2001), albeit at the lower limit. We accept both galaxies as SBF-confirmed Fornax cluster members, but assign IM1_2_2_LSB1 an intermediate membership flag $=1.3$ due to its low $S / N$ in the SBF measurement (see Table 1).

None of the other new candidates had a measurable SBF signal. It must therefore be checked whether among them may be any bona-fide SBF background galaxy. From Fig. 6 it is clear that, apart from the two candidates with SBF signal, six more have central surface brightnesses $<24.5 \mathrm{mag} / \operatorname{arcsec}^{2}$, which was the approximate SBF detection limit for the entire sample of $\mathrm{dE}$ candidates from Paper II. Three out of those six are detected in the central dithered IMACS pointing which only had $1800 \mathrm{~s}$ total exposure time. Two of the galaxies, IM1_2_6_LSB1 and IM1_1_7_LSB1, are indeed detected in only one of the two dithered exposures of a $900 \mathrm{~s}$ integration time. This substantial reduction in integration time by a factor of 2-4 compared to the other fields results in a brighter limiting magnitude for SBF measurement by 1-2 mag (see Paper I), thus explaining the absence of an SBF signal. Two more galaxies IM1_6_LSB1 and IM1_6_LSB2 had very large PSF $F W H M \simeq 1.6^{\prime \prime}$, since 
Table 2. Summary of the SBF data for the two new dE candidates from Sect. 5 with detectable SBF signal. See Mieske et al. (2006b, Paper III) and Sect. 3 of this paper for details on the SBF measurement procedure.

\begin{tabular}{r|rrrrrr}
\hline \hline Name & $\bar{m}_{I}$ & $(V-I)_{0, \mathrm{SBF}}$ & $S / N$ & $B G$ & $\Delta_{\mathrm{GC}}$ & $(m-M)$ \\
\hline IM4_2_LSB1 & $28.28 \pm 0.37$ & 0.932 & 5.0 & 0.36 & 0.01 & $31.00 \pm 0.39$ \\
IM1_2_2_LSB1 & $28.13 \pm 0.37$ & 0.920 & 1.9 & 0.12 & 0.02 & $30.91 \pm 0.39$ \\
\hline
\end{tabular}

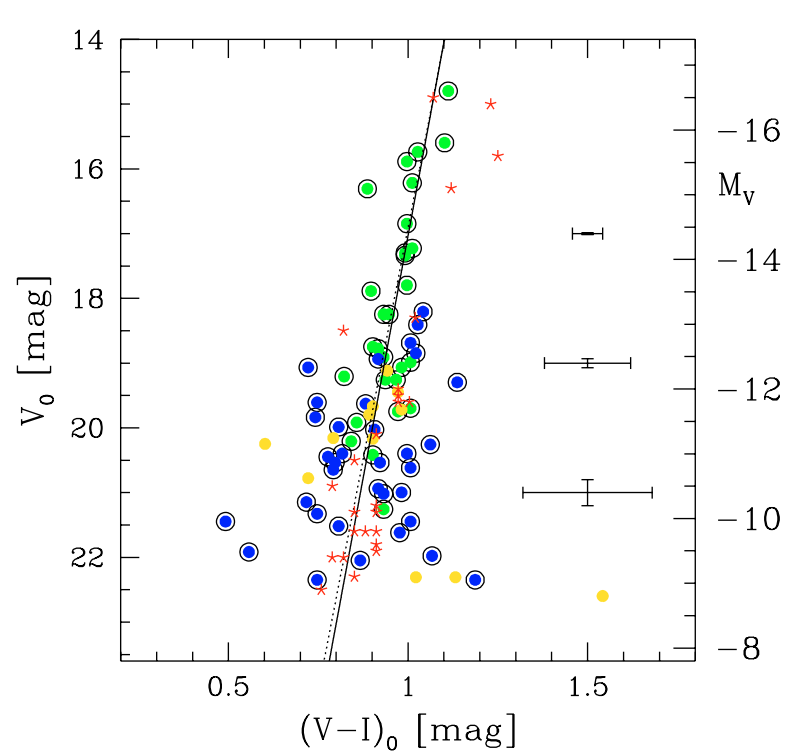

Fig. 9. Colour-magnitude diagram (CMD) of the sources from Fig. 6. Typical error bars are indicated. The magnitudes and colours were redenning corrected using Schlegel et al. (1998). The $V$ magnitude is from the IMACS data, while $(V-I)$ is adopted as the mean of the WFCCD and IMACS values. Adopting this mean reduces the colour scatter by about $35 \%$ as compared to the single IMACS colour: the colour scatter for the IMACS values is $0.15 \mathrm{mag}$, while that of the mean (and also the WFCCD colour) is about $0.11 \mathrm{mag}$. The solid line is a fit to the data points applying a $3 \sigma$ clipping. The dotted line - which is practically on top of the solid line - indicates the colour-magnitude relation derived from the WFCCD photometry of all dE candidates (Hilker et al. 2003, Paper II). The (red) Local Group data points for $M_{\mathrm{V}}<-13$ mag are proper $(V-I)$ measurements from Mateo et al. (1998), while for $M_{\mathrm{V}}>-13$ mag the $(V-I)$ values are estimated from their $[\mathrm{Fe} / \mathrm{H}]$ values (Grebel et al. 2003). For this transformation we applied equation (4) from Kissler-Patig et al. (1998), and adopted an additional zero point shift of -0.055 mag. This shift was the difference between the transformed and measured $(V-I)$ values for the five Local Group dEs with $M_{\mathrm{V}}<-13$ mag that do have a measured $(V-I)$.

located in a part of the field of view with strong image distortion (see Fig. 8). This leaves one single object, IM7_6_LSB1, as a galaxy with reasonably good seeing and long $I$-band integration time. Figure 8 shows that this galaxy is very compact, such that it yields too few independent data points for SBF sampling. Furthermore, this galaxy has the bluest colour of all new dE candidates (see next section and Fig. 9). It may therefore be a background blue compact dwarf or unresolved background spiral. We have assigned this galaxy an intermediate cluster membership flag of 2.3 (see Table 1).

\section{The revised photometric properties of Fornax early-type dwarf galaxies}

In this section we discuss the photometric properties of the early type Fornax dwarf galaxy population based on the IMACS photometry. We include in our analysis those galaxies from Table 1 with membership flag $<2.5$. They are the ones directly classified as cluster members from SBF (Sect. 3), plus those that are probable cluster members from our revised morphological assessment (Sect. 4), plus those that were newly discovered in the IMACS data (Sect. 5).

Total galaxy magnitudes were derived with the IMACS data as for the WFCCD data by curve-of-growth analyses using the IRAF task ELLIPSE. Along each isophote fitted by ELLIPSE, a $3 \sigma$ clipping algorithm was applied to reject contaminating sources. The sky level was adjusted individually for every galaxy in the course of the curve-of-growth analysis. Total magnitudes were derived by summing up the fitted intensities up to a cutoff radius determined by the curve-of-growth analysis. Central surface brightnesses were determined by fitting an exponential function to the surface brightness profile, excluding the nuclear regions of $\mathrm{dE}, \mathrm{Ns}$.

Colours from the IMACS data were derived as the difference between $V$ and $I$ magnitudes within an aperture of $4^{\prime \prime}$ radius, exactly like for the WFCCD data. We adopted the mean of the WFCCD and IMACS values as the final galaxy colour, given that the scatter in the colour-magnitude plane reduces by $35 \%$ when doing so. Zero-point colour offsets between both photometry sets are negligible: the mean colour difference between IMACS and WFCCD data is $-0.006 \pm 0.026 \mathrm{mag}$.

It is worth going through the procedure applied to obtain a realistic colour error for each galaxy. The first step was to estimate a global uncertainty of the sky background determination for the IMACS data. The corresponding colour error then is robust in a relative sense such that galaxies with fainter surface brightness have correspondingly larger colour errors. For the global sky background uncertainty in the IMACS data, we adopted the rms scatter between two sets of estimates: first, the background obtained "manually" from the curve-of-growth analysis. Second, the sky background obtained from subtracting a SExtractor sky map off the galaxy image, which itself had previously been cleaned of all objects using a SExtractor object map.

To also obtain a realistic colour error in an absolute sense, we re-scaled the estimated global sky background uncertainty such that the resulting average colour error equals the RMS scatter between the IMACS and WFCCD colours. The colour errors derived in this way were on average around $0.10 \mathrm{mag}$, with a broad range between 0.02 mag for the highest surface brightness galaxies and almost 0.40 mag for the faintest ones (see Table 1).

For $\mu_{\mathrm{V}, 0}$ and $M_{V, 0}$, we used only the IMACS values, given that the scatter of the mean values in the magnitude-surface brightness plane is marginally larger than the scatter of the IMACS values alone. Errors in $\mu_{\mathrm{V}, 0}$ and $M_{V, 0}$ were derived from the uncertainty in the sky background determination in the IMACS data, as outlined in the previous paragraph. The resulting errors are given in Table 1 . We finally note that the IMACS $\mu_{\mathrm{V}, 0}$ and $M_{V, 0}$ values are on average about $0.1 \mathrm{mag}$ fainter than the WFCCD values. This is because the higher resolution IMACS data allowed better masking of contaminating point sources close to the galaxy centres, especially for the lower surface brightness galaxies. 


\subsection{Magnitude-surface brightness relation}

In Fig. 6 we show the IMACS magnitude-surface brightness plot, with typical error bars indicated. Data for LG dEs are shown for comparison. We fit the following relation between central surface brightness $\mu_{\mathrm{V}, 0}$ and absolute magnitude $M_{V, 0}$, assuming $(m-M)=31.39 \mathrm{mag}$ and applying a $3 \sigma$ clipping algorithm $^{2}$ :

$\mu_{\mathrm{V}, 0}=32.32( \pm 1.12)+0.681( \pm 0.040) \times M_{V, 0}$.

Assuming the same slope, the relation defined by the LG dEs has an offset of $0.75 \pm 0.15$ mag towards brighter $\mu_{\mathrm{V}, 0}$. In other words, the Fornax dEs in our sample are about $40 \%$ larger than the LG dEs at equal central surface brightnesses. There have also been indications of such a size difference for Virgo cluster dwarfs: Caldwell \& Armandroff (2000) and Caldwell (2005) report on the discovery of a large population of very low surface brightness $\mathrm{dEs}$ in the Virgo cluster whose sizes extend to significantly higher values than known for LG dEs.

An offset in mean size may be explained within a scenario where tidal forces disrupt the smallest and least massive dEs more effectively in denser environments like the Fornax or Virgo cluster than in the LG (e.g. Hilker et al. 1999c). However, Fig. 7 suggests that the Fornax cluster also hosts an overabundance of larger dEs, in addition to an underabundance of smaller ones. Indeed, the surface brightness distribution of LG and Fornax totally agree with each other once a simple offset to the LG values is applied, see Fig. 7. One may therefore speculate that tidal heating (e.g. Valluri 1993; Das \& Jog 1995) could be enhancing the internal energy of dEs in the Fornax cluster more than for LG dEs. This is conceivable since tidal heating effects are expected to be more pronounced in denser environments (Valluri 1993), as is the Fornax cluster in comparison with the LG. It remains to be clarified whether the vastly dark-matterdominated faint dEs could indeed be sufficiently affected by the cluster tidal field.

Another mechanism that may in principle cause environmental differences among galaxy populations is re-ionization (e.g. Dekel \& Woo 2003; Moore et al. 2006). It is reasonable to assume that those galaxy halos that collapsed in the densest regions of the universe collapsed very early and consequently had more time to form stars before re-ionization than halos in less dense regions of the universe. This may have led to less centrally concentrated stellar halos in galaxies located in high density environments like Fornax. However, Grebel \& Gallagher (2004) rule out that the star formation histories of most LG dwarfs were decisively influenced by re-ionization. Along these lines, Ricotti et al. (2002) also argue that the star formation histories of low mass dark-matter halos are almost independent of the external radiation field and mostly influenced by radiative feedback.

A general concern about the comparison between the LG and other environments is that the LG sample may be highly incomplete. In that context it is interesting to note that the latest discoveries of new faint LG members with $M_{\mathrm{V}} \simeq-9$ mag (e.g. Armandroff et al. 1999; Whiting et al. 1999; Zucker et al. 2004) are restricted to rather small scale sizes. This may indicate that the LG dE sample is more incomplete at smaller than at larger galaxy sizes. The fact that large scale stellar overdensities like the Sagittarius and Monoceros streams are quite

\footnotetext{
2 The fit errors were derived from random resampling of the data points within their measured scatter. Given that the scatter of values in Fig. 6 is much larger than the measurement uncertainty in both $V_{0}$ and $\mu_{\mathrm{V}, 0}$, we do not error weight the data points in the fit.
}

readily detected in all-sky surveys (Ibata et al. 2001; Majewski et al. 2004; Peñarrubia et al. 2005) seems to support this impression.

It is clear that more work still needs to be done regarding this subject: regarding both a comparison of structural parameters of LG dwarfs with their counterparts in nearby clusters and the theoretical framework of dwarf galaxy formation.

\subsection{Colour-magnitude relation}

In Fig. 9 we show the colour-magnitude diagram of the same sources as in the magnitude-surface brightness plot in Fig. 6, with typical error bars indicated. Data for LG dEs are also shown for comparison. We fit the following relation between colour $(V-I)_{0}$ and absolute magnitude $M_{V, 0}$, applying a $3 \sigma$ clipping algorithm ${ }^{3}$

$$
(V-I)_{0}=0.52( \pm 0.07)-0.033( \pm 0.004) \times M_{V, 0} .
$$

The colours of Fornax dEs agree reasonably well with the values for Local Group dEs except for the brightest few galaxies, indicating overall similar self-enrichment histories for both populations. There is a marginal shift of $0.032 \pm 0.014$ mag towards redder colours for the LG data. However, it must be noted that the LG dE colours for $M_{\mathrm{V}}>-13$ mag are not direct measurements but are rather estimated from their $[\mathrm{Fe} / \mathrm{H}]$ values (see caption of Fig. 9). When restricting the consideration to $M_{\mathrm{V}}<-13$ mag the colour difference is substantially larger with $0.14 \pm 0.05$ mag. The nominal slope of the colour-magnitude correlation for the LG dEs is $-0.047 \pm 0.005$, about $40 \%$ higher than for the Fornax dEs at $2.2 \sigma$ significance. We note that these differences are consistent with a higher self-enrichment efficiency among LG dEs than in Fornax dEs - especially in the brighter luminosity regime. It is an interesting speculation that the more compact light distribution of LG dEs (see previous sub-section) may be part of the reason for such a stronger self-enrichment, provided that light traces mass in a similar manner in both environments. Both a stronger gravitational potential and a higher gas density may have supported self-enrichment in Local Group dEs more than in Fornax dEs. The smaller sizes of Local Group dEs and the (still preliminary) colour differences between LG and Fornax dEs could therefore be two sides of the same medal.

\subsection{Galaxy luminosity function}

In Fig. 10 we show the Fornax dE GLF, as derived from scaling the Paper II GLF with the fraction of galaxies that are confirmed as cluster members in this paper. Note that the inclusion of the new candidates from Sect. 5 raises this fraction above unity in some magnitude bins.

The denominator of this fraction is the number of galaxies per magnitude bin from Paper II that enter in the calculation of the Paper II LF. Note that these are only those that lie within the $2 \sigma$ limits of both the colour and surface-brightness magnitude relation defined by the entire sample. This restriction had been applied in Paper II to reduce the effect of contaminators to the sample, given the limited morphological selection potential of the WFCCD data especially in the parameter space defined by LG dEs. The IMACS data largely removes this restriction by virtue of its improved spatial resolution. For the nominator we hence adopt the number of confirmed cluster member candidates

\footnotetext{
3 For this fitting we weighted each data point by its colour error, given that the scatter in the CMD is consistent with being entirely created by colour measurement errors.
} 


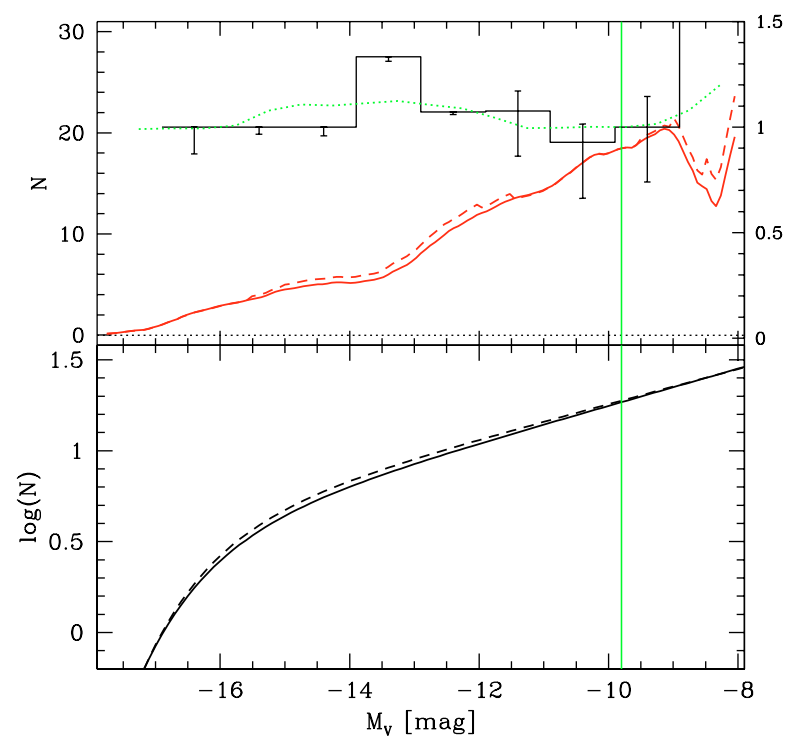

Fig. 10. This figure shows how the Fornax cluster galaxy luminosity function from Hilker et al. (2003, Paper II) is re-assessed in the present paper. The vertical (green) line indicates the 50\% detection limit from Paper II, which is also the faint limit for fitting the faint end slope $\alpha$. Top panel: the solid (red) line indicates a smoothed representation of the Fornax GLF from Paper II. The solid histogram gives the fraction of correct cluster member candidate identifications determined in Sects. 3 to 4, augmented by the inclusion of the new candidate cluster members from Sect. 5. Note that this inclusion of members that were undetected in Paper II makes the fraction larger than 1 in some bins. The (green) dotted line is a smoothed representation of the histogram. The dashed red line indicates the corrected Fornax GLF, i.e. the product of the Paper II LF with the dotted line. See Sect. 6.3 for more details. Bottom panel: solid line: 1-component fit to the Fornax-GLF from Paper II. Dashed line: 1-component fit to the corrected Fornax GLF. The respective faint end slopes are $\alpha=-1.10$ for the Paper II LF and $\alpha=-1.09$ for the corrected LF.

- i.e. those with flag $<2.5$ in Table $1-$ without applying any cut. We do however exclude those five new $\mathrm{dE}$ candidates that were outside the WFCCD FOV.

Applying a one component Schechter function fit to the resulting dwarf galaxy luminosity function down to $M_{\mathrm{V}}=$ $-9.8 \mathrm{mag}$ (the 50\% WFCCD completeness limit), the faint end slope is $\alpha=-1.10 \pm 0.10$ both for the Paper II GLF and for the corrected GLF (see Fig. 10). Note that the Paper II GLF and hence also the corrected GLF is incompleteness corrected. The nominal slope difference is below 0.01 . Also the inclusion of the five new $\mathrm{dE}$ candidates outside the WFCCD FOV does not alter the slope by more than 0.01 . After restricting the fit to the very faint end $M_{\mathrm{V}}>-13.5 \mathrm{mag}$ (the onset of the $\mathrm{dSph}$ regime, see Grebel et al. 2003), the values are $\alpha=-1.06$ for the Paper II GLF and $\alpha=-1.04$ for the corrected GLF. The corrected slope is only marginally shallower than the value from Paper II, much smaller than the statistical uncertainty of \pm 0.10 .

It is comforting that the faint end slope of the Fornax dwarf GLF remains in the range -1.1 to -1.0 when going from the early studies of Ferguson \& Sandage (1988) and Ferguson (1989) to the present paper that samples the GLF to about 3 mag fainter. We are therefore confident that this value is robust and not very biased by systematic effects. Morphological cluster membership assignment in Fornax apparently is very reliable, provided that the image resolution is sufficient.

It is well known that such a shallow faint end slope sharply contradicts the much steeper value predicted for the mass function of $\Lambda C D M$ halos (e.g.Kauffman et al. 2000; Moore et al. 1999). Possible reasons for that discrepancy include the accretion scenario (e.g. Hilker et al. 1999c; Côté et al. 1998), where dwarf galaxies that fall into the cluster centres are tidally disrupted, hence contributing to forming the extended $\mathrm{cD}$ halos of the most massive cluster galaxies like NGC 1399 in Fornax. The presence of ultra-compact dwarf galaxies (UCDs) in the central Fornax cluster (Hilker et al. 1999b; Drinkwater et al. 2003) may be a signpost of these tidal interactions, given that some but possibly not most - of them may be tidally stripped dE,Ns (Bekki et al. 2003; Mieske et al. 2006a). Another possibility is that the low surface brightness dEs that we see nowadays originate from tidally-stripped and originally much more massive dark matter halos (Stoehr et al. 2002; Kravtsov et al. 2004), while the lower-mass halos have not been able to maintain condensed gas to form stars. However, Kazantzidis et al. (2004) argue that observed velocity dispersion profiles of Local Group $\mathrm{dE}$ exclude such very massive progenitors, indicating that tidal stripping can only be a part of the picture. More work on the theoretical side is certainly needed to better understand the mechanisms that create and destroy dark matter dominated stellar systems at the low mass end (e.g. Hayashi et al. 2003; Moore et al. 2006).

\section{Summary and conclusions}

In this paper we have presented a photometric analysis of the early-type dwarf galaxy population $\left(M_{\mathrm{V}}>-17 \mathrm{mag}\right)$ in the central Fornax cluster, covering the central square degree and slightly beyond. This analysis is based on wide field imaging in $V$ and $I$ using the instrument IMACS mounted at the $6.5 \mathrm{~m}$ Walter Baade Magellan telescope at Las Campanas Observatory, Chile. The pixel scale of $0.2^{\prime \prime}$ and $0.8^{\prime \prime}$ median seeing $F W H M$ enabled us to efficiently resolve $\mathrm{LG} \mathrm{dE}$ analoga down to $M_{\mathrm{V}} \simeq$ -10 mag. We used these data to follow up a previous imaging survey of our group (Hilker et al. 2003, Paper II) that had a four times larger pixel scale and seven times smaller light collecting area.

We summarise our main results as follows:

1. We confirm the cluster member status for 28 candidate dEs from Paper II in the magnitude range $-16.6<M_{\mathrm{V}}<$ -10.1 mag by means of $I$-band surface brightness fluctuation $(\mathrm{SBF})$ measurements.

2. We re-assess the morphological classification of 51 more candidate dEs from Paper II in the range $-13.2<M_{\mathrm{V}}<$ $-8.6 \mathrm{mag}$ based on the much improved imaging resolution. Of these, $2 / 3$ retain their smooth dE-like appearence on our images, hence are confirmed as probable cluster members. About $1 / 3$ are re-classified as probable background galaxies, most of which have sizes close to the resolution limit of the data from Paper II. We cannot confirm the status of a background galaxy by means of SBF measurement. This is because the galaxies without a measurable SBF signal are about the same as or fainter than the limiting surface brightness for SBF detection at the Fornax cluster distance.

3. We find 12 new dE candidates in the range $-12.3<M_{\mathrm{V}}<$ -8.8 mag whose intrinsic sizes are close to the resolution limit of the data from Paper II. These detections result from a search for low surface brightness features trimmed to detect analogs of Local Group dEs with $M_{\mathrm{V}}>-12.5$ mag. Two of the new candidates can be confirmed via SBF measurement. 
4. We investigate the surface brightness-magnitude relation for the joint sample of confirmed dE candidates from items (1) to (3). We derive:

$\mu_{\mathrm{V}, 0}=32.32+0.681 \times M_{V, 0}$.

We find that the Fornax dEs are shifted by $0.75 \pm 0.15 \mathrm{mag}$ towards fainter $\mu$ at a given luminosity compared to Local Group dEs; i.e., Fornax dEs are about $40 \%$ larger than Local Group dEs. We briefly discuss possible reasons for this difference.

5. We investigate the colour-magnitude relation for the same sample of Fornax dEs and find the following fit:

$$
(V-I)_{0}=0.52-0.033 \times M_{V, 0} .
$$

The derived slope is slightly shallower than estimated for Local Group dEs, mainly driven by the fact that Local Group dEs with $M_{\mathrm{V}}<-13$ mag appear significantly redder than their Fornax counterparts of same luminosity. We indicate that in the context of self-enrichment this may be expected from the fact that Local Group dEs have a more compact stellar body than Fornax dEs.

6. We re-scale the Paper II dwarf galaxy luminosity function (GLF) in Fornax as a function of magnitude by the fraction of confirmed candidate dEs derived in items (1) to (3). A one-component Schechter fit of the corrected GLF down to $M_{\mathrm{V}}=-9.8$ mag yields a faint end slope $\alpha$ differing by less than 0.01 from the value $\alpha=-1.10 \pm 0.10$ derived in Paper II. When restricting the fit to $-13.5<M_{\mathrm{V}}<-10 \mathrm{mag}$, the slope is $\alpha=-1.04$ for the corrected GLF and -1.06 for the Paper II GLF. Our results confirm a very shallow faint end slope for the Fornax dwarf galaxy luminosity function, in agreement with early estimates in the reference study of Ferguson \& Sandage (1988). We briefly discuss this finding in the context of structure formation models.

We conclude that for nearby clusters such as Fornax, the SBF method is a powerful tool for extending the limit of cluster membership determination down to the regime where the faint end slope $\alpha$ dominates the shape of the GLF. Our predictions for the potential of the SBF method (Mieske et al. 2003, Paper I) are confirmed by the results of this paper. We can also conclude that for the Fornax cluster case, the morphological cluster membership assignment is very efficient, provided that the image resolution is substantially better than the expected galaxy size. Future targets for studies like this one include the Virgo cluster or other nearby galaxy groups within about $20 \mathrm{Mpc}$.

Acknowledgements. We thank the staff at Las Campanas Observatory for their friendly and very efficient support during the execution of the imaging runs. S.M. acknowledges support by DFG project HI 855/1 and DAAD Ph.D. grant Kennziffer D/01/35298. L.I. was supported by the FONDAP "Center for Astrophysics". C.M.d.O. would like to thank the Universitätssternwarte München and the Max Planck Institut für Extraterretrische Physik for their hospitality. C.md.O. acknowledges support from FAPESP (proyeto temático 2001/07342-7).

\section{References}

Andreon, S., Cuillandre, J. C., Puddu, E., \& Mellier, Y. 2006, MNRAS, 372, 60 Armandroff, T. E., Jacoby, G. H., \& Davies, J. E. 1999, AJ, 118, 1220

Bekki, K., Couch, W. J., Drinkwater, M. J., \& Shioya, Y. 2003, MNRAS, 344, 399

Bertin, E., \& Arnouts, S. 1996, A\&AS, 117, 393

Caldwell, N. 1987, AJ, 94, 1116

Caldwell, N., \& Armandroff, T. A. 2000, 197th AAS Meet., \#106.05, Bull. Am. Astron. Soc., 32, 1579

Caldwell, N. 2005, Near-fields cosmology with dwarf elliptical galaxies, Proceedings of IAU colloquium, held 14-18 March, Switzerland, ed. H. Jerjen, \& B. Binggeli (Cambridge: Cambridge University Press), 198, 285

Côté, P., Marzke, R. O., \& West, M. J. 1998, ApJ, 501, 554

Das, M., \& Jog, C. J. 1995, ApJ, 451, 167

Dekel, A., \& Woo, J. 2003, MNRAS, 344, 1131 ApJ, 438, L13

Drinkwater, M. J., Gregg, M. D., Holman, B. A., \& Brown, M. J. I. 2001, MNRAS, 326, 1076

Drinkwater, M. J., Gregg, M. D., Hilker, M., et al. 2003, Nature, 423, 519

Ferguson, H. C. 1989, AJ, 98, 367

Ferguson, H. C., \& Sandage, A. 1988, AJ, 96, 1520

Freedman, W. L., Madore, B. F., Gibson, B. K., et al. 2001, ApJ, 553, 47

Grebel, E. K., Gallagher, J. S., \& Harbeck, D. 2003, AJ, 125, 1926

Grebel, E. K., \& Gallagher, J. S. 2004, ApJL, 610, 89

Hayashi, E., Navarro, J. F., Taylor, J. E., Stadel, J., \& Quinn, T. 2003, ApJ, 584, 541

Hilker, M., Kissler-Patig, M., Richtler, T., Infante, L., \& Quintana, H. 1999a, A\&AS, 134, 59

Hilker, M., Infante, L., Vieira, G., Kissler-Patig, M., \& Richtler, T. 1999b, A\&AS, 134, 75

Hilker, M., Infante, L., \& Richtler, T. 1999c, A\&AS, 138, 55

Hilker, M., Mieske, S., \& Infante, L. 2003, A\&A, 397, L9 (Paper II)

Ibata, R., Irwin, M., Lewis, G. F., \& Stolte, A. 2001, ApJ, 547, L133

Infante, L., Mieske, S., \& Hilker, M. 2003, Ap\&SS, 285, 87

Kauffmann, G., \& Haehnelt, M. 2000, MNRAS, 311, 576

Jenkins, A., Frenk, C. S., White, S. D. M., et al. 2001, MNRAS, 321, 372

Kambas, A., Davies, J. I., Smith, R. M., Bianchi, S., \& Haynes, J. A. 2000, AJ, 120,1316

Kazantzidis, S., Mayer, L., Mastropietro, C., et al. 2004, ApJ, 608, 663

Kissler-Patig, M., Brodie, J. P., Schroder, L. L., et al. 1998, AJ, 115, 105

Kravtsov, A. V., Gnedin, O. Y., \& Klypin, A. A. 2004, ApJ, 609, 482

Majewski, S. R., Kunkel, W. E., Law, D. R. et al. 2004, AJ, 128, 245

Mateo, M. L. 1998, ARA\&A, 36, 435

Mieske, S., Hilker, M., \& Infante, L. 2003, A\&A, 403, 43 (Paper I)

Mieske, S., Hilker, M., \& Infante, L. 2004, A\&A, 418, 445

Mieske, S., Hilker, M., Infante, L., \& Jordán, A. 2006a, AJ, 131, 2442

Mieske, S., Hilker, M., \& Infante, L. 2006b, A\&A, 458, 1013 (Paper III)

Moore, B., Ghigna, S., Governato, F., et al. 1999, ApJ, 524, L19

Moore, B., Diemand, J., Madau, P., Zemp, M., \& Stadel, J. 2006, MNRAS, 368, 563

Peñarrubia, J., Martínez-Delgado, D., Rix, H. W., et al. 2005, ApJ, 626, 128 Phillipps, S., Disney, M. J., Kibblewhite, E. J., \& Cawson, M. G. M. 1987, MNRAS, 229, 505

Ricotti, M., Gnedin, N. Y., \& Shull, J. M. 2002, ApJ, 575, 49

Schlegel, D. J., Finkbeiner, D. P., \& Davis, M. 1998, ApJ, 500, 525

Stoehr, F., White, S. D. M., Tormen, G., \& Springel, V. 2002, MNRAS, 335, L84

Tanaka, M., Kodama, T., Arimoto, N., et al. 2005, MNRAS, 362, 268

Tonry, J. L., \& Schneider, D. P. 1988, AJ, 96, 807

Tonry, J. L., Dressler, A., Blakeslee, J. P., et al. 2001, ApJ, 546, 681

Trentham, N., \& Tully, R. B. 2002, MNRAS, 335, 712

Trentham, N., Sampson, L., \& Banerji, M. 2005, MNRAS, 357, 783

Valluri, M. 1993, ApJ, 408, 57

Whiting, A. B., Hau, G. K. T., \& Irwin, M. J. 1999, AJ, 118, 2767

Zucker, D. B., Kniazev, A. Y., Bell, E. F., et al. 2004, ApJ, 612, L121 